\title{
Liposomal Drug Delivery System - A Concise Review
}

\author{
Sakib M Moinuddin ${ }^{1 *}$, Irfan Sajid ${ }^{2}$ and Md. Hasanul Arif ${ }^{3}$ \\ ${ }^{1}$ Guilin Pharmaceutical Co., Ltd., R\&D Center, Guangxi, China \\ ${ }^{2}$ Department of Pharmaceutical Sciences, North South University, Bangladesh \\ ${ }^{3} R \& D$ Department, Orion Pharmaceutical Company Limited, Dhaka, Bangladesh \\ *Corresponding Author: Sakib M Moinuddin, E-mail: sa12kib@yahoo.com
}

Received: November 15, 2021

Published: December 16, 2021

(C) All rights are reserved by Sakib M

Moinuddin., et al.

\begin{abstract}
Liposomes are microscopic self-assembling unilamellar or multilamellar vesicles made up of phospholipid bilayer. Both the hydrophilic and hydrophobic drugs can be attached to the lipid bilayer of liposomes and can show their efficacy in the target cell of the human body. After discovering the liposome by Bangham., et al. and his colleagues, this drug delivery system gained much popularity in every aspect of the pharmaceutical dosage form. Recently, the liposomal drug delivery system has been commercially manufactured and applied in a variety of ailments, including black fungal infection of post-COVID 19, cancer, analgesics, vaccines, and photodynamic therapy, etc. This review tersely described the liposomal formation, classification, characterization, and the overview of marketed drugs.
\end{abstract}

Keywords: Liposome; Phospholipids; Black Fungus; COVID 19

\section{Introduction}

Recently, more than $40 \%$ of marketed drugs and drug discovery pipelines 70 to $90 \%$ are suffering poor aqueous solubility and permeability problems [1,2]. The computerized combinatorial chemistry has led to discovering new drug entities by potential therapeutic action on the target cell. These poor solubilities and permeability can lead to low bioavailability $[3,4]$. The anticancer or chemotherapeutic agents are highly cytotoxic to the malignant cell, equally damaging the normal cells. For this reason, drug substances need a specific formulation that can specifically bind with the target disease site without damaging the normal cell. At present, formulation scientists have attempted several techniques, including nanoparticles, microparticles, and liposomes, for the targeted drug delivery to the specific disease site.

The liposome is a spherical-shaped vesicle that consists of one or more phospholipid bilayers. Notably, the liposome is a carrier for drugs or targeted molecules. Interestingly, liposome formulation can be encapsulated by both hydrophilic and lipophilic drugs, i.e., biological classification system (BCS) class i, ii, iii, and iv drugs, and applied to the disease site of the body. This system is made of colloidal size with a range of 0.01 to $5.0 \mu \mathrm{m}$ in diameter [5].

In this review, we provide a concise picture of the liposomebased products on the market. In addition, we mentioned liposomal formation, classification, and characterization.

\section{Formation of liposome}

A liposome is a potential carrier of the hydrophilic and lipophilic drug substance. Figure 1 represents the liposome of sphericalshaped phospholipid bilayers. The hydrophobic drug substance is loaded on the hydrophobic part, and the hydrophilic drug substance is loaded on the hydrophilic portion of the liposome (Figure 1).

\section{Commercial liposomal drug delivery}

Currently, several pharmaceutical agents, including vaccine, antiviral, anticancer drugs, proteins, and macromolecules, are en- 


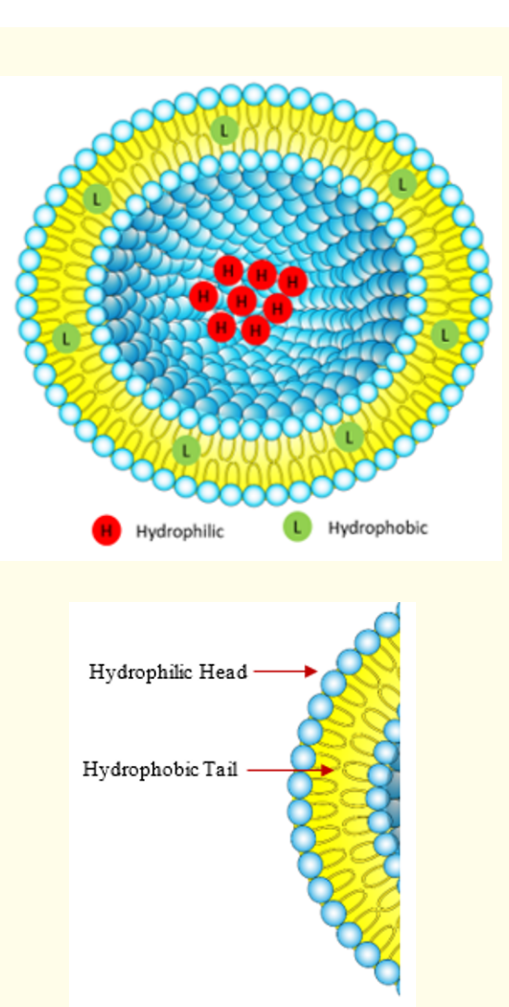

Figure 1: Liposomal drug delivery.

capsulated commercially in liposomal form [6] (Table 1). Recently liposomal injection amphotericin B was successfully applied in post-COVID 19 infected black fungus [7-9]. Liposomal amphotericin $B$ injection is very effective against black fungal disease. The black fungus infects the lungs, stomach, intestine, skin, sinuses, and brain; however, this disease is rare, and thousands of infected cases were reported during the second wave of COVID 19 in India and a few reported cases in Bangladesh [8,10].

\begin{tabular}{|c|c|c|c|c|}
\hline Drug Name & Trade Name & Year & Manufacturer & Indications \\
\hline \multirow{3}{*}{$\begin{array}{l}\text { Amphoteri- } \\
\operatorname{cin} \text { B }\end{array}$} & Abelcet $^{\circledR}$ & 1995 & Enzon & \multirow{3}{*}{$\begin{array}{c}\text { Fungal } \\
\text { infection }\end{array}$} \\
\hline & Amphotec $^{\circledR}$ & 1996 & $\begin{array}{c}\text { Ben Venue } \\
\text { Laboratories, } \\
\text { Inc }\end{array}$ & \\
\hline & Ambisome $^{\mathrm{TM}}$ & 1997 & Gilead Sciences & \\
\hline Daunorubicin & DaounoXome ${ }^{\mathrm{TM}}$ & - & Gilead Sciences & $\begin{array}{l}\text { Kaposi's } \\
\text { sarcoma }\end{array}$ \\
\hline \multirow{4}{*}{ Doxorubicin } & Doxil $^{\mathrm{TM}}$ & 1995 & Ortho Biotech & \multirow{4}{*}{\begin{tabular}{|} 
Refractory \\
Kaposi's \\
sarcoma, \\
recurrent \\
breast cancer, \\
and ovarian \\
cancer
\end{tabular}} \\
\hline & Caelyx $^{\mathrm{TM}}$ & 1995 & Schering-Plough & \\
\hline & Myocet $^{\circledR}$ & 2000 & Zeneus & \\
\hline & Lipo-Dox ${ }^{\circledR}$ & - & TTY BIOPHARM & \\
\hline
\end{tabular}

\begin{tabular}{|c|c|c|c|c|}
\hline Verteporfin & Visudyne $^{\circledR}$ & 2000 & $\begin{array}{c}\text { Valeant Canada } \\
\text { LP }\end{array}$ & $\begin{array}{l}\text { Age-related } \\
\text { macular } \\
\text { degeneration, } \\
\text { pathologic } \\
\text { myopia, and } \\
\text { ocular } \\
\text { Histoplasmo- } \\
\text { sis } \\
\end{array}$ \\
\hline Cytarabine & DepoCyt $^{\circledR}$ & 1999 & $\begin{array}{l}\text { Pacira (formerly } \\
\text { SkyePharma) }\end{array}$ & $\begin{array}{l}\text { Neoplastic } \\
\text { meningitis } \\
\text { and } \\
\text { lymphoma- } \\
\text { tous } \\
\text { meningitis }\end{array}$ \\
\hline Cisplatin & Lipoplatin ${ }^{\circledR}$ & 2007 & Regulon, Inc & $\begin{array}{c}\text { Epithelial } \\
\text { malignancies }\end{array}$ \\
\hline $\begin{array}{l}\text { Morphine } \\
\text { sulfate }\end{array}$ & DepoDur $^{\circledR}$ & 2004 & $\begin{array}{c}\text { SkyePharma, } \\
\text { Endo }\end{array}$ & $\begin{array}{l}\text { Postoperative } \\
\text { pain following } \\
\text { major surgery }\end{array}$ \\
\hline
\end{tabular}

Table 1: List of marketed drugs.

Besides post-COVID 19 treatment, liposomal drug delivery is also applied in cancer therapy, different types of fungal disease, analgesics, viral vaccines, and photodynamic therapy [6]. The liposomal dosage form is also involved in pulmonary arterial hypertension and lung disease. Furthermore, several researchers are working on the liposomal drug delivery systems to develop dry powder inhaler (DPI), organ-on-chip or nanochip, and invasive treatment.

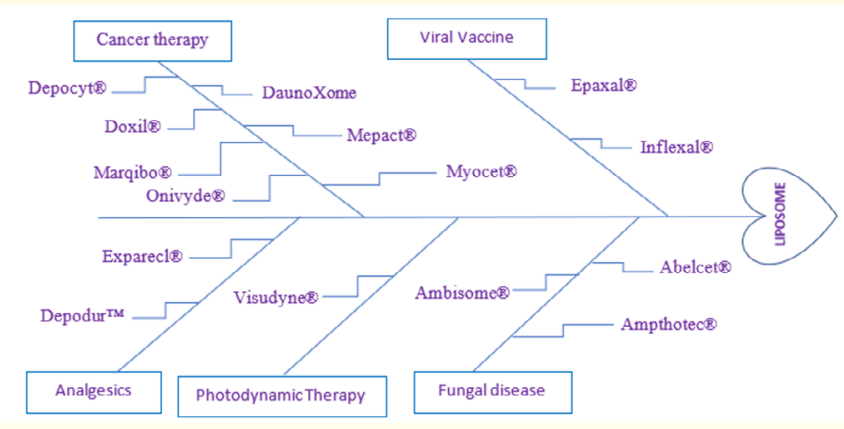

Figure 2: Different therapeutic are covered by liposomal drug [6].

\section{Classification of liposome}

Liposome classification is based on the size, the number of phospholipid bilayers, composition, and drug delivery mechanism 
$[11,12]$. However, based on the size and number of the phospholipid bilayers, the liposomes are divided into two classes named one phospholipid and several phospholipids. Both categories can further be subdivided into several groups. Moreover, liposomes are divided into several groups depending on the composition, including conventional liposomes, fusogenic liposomes, $\mathrm{pH}$-sensitive liposomes, cationic liposomes, long-circulating liposomes, and immune liposomes [12].

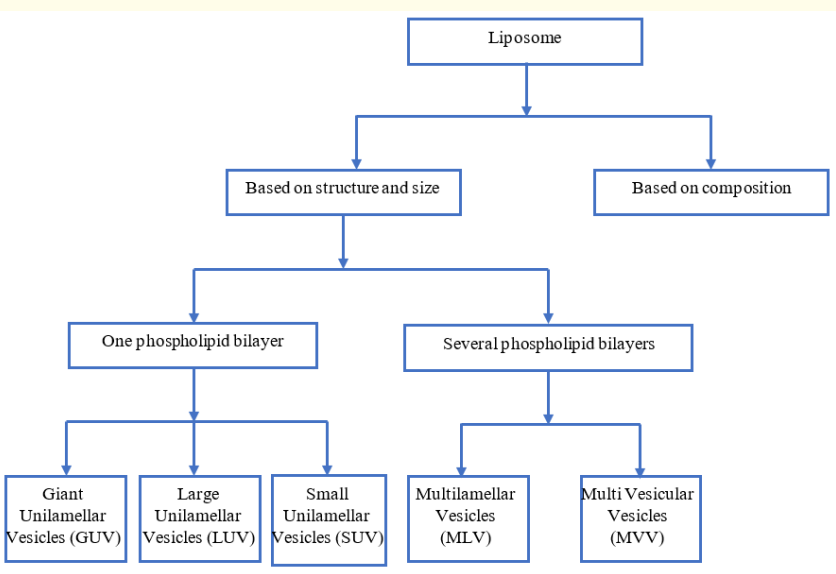

Figure 3: Schematic diagram of liposomal classification.

\section{Preparation of liposomes}

In 1961, the lipid-based vesicles were reported by Bangham., et al. and his coworker [13]. After that, plenty of liposome preparation has been utilized, including lipid hydration, ethanol injection, freeze-drying, reverse phase evaporation, etc. In the conventional methods, the lipid is initially dissolved in an organic solvent and then dried by a rotary solvent evaporation technique. The lipids are dispersed in an aqueous solution, and then the resulting liposomes are purified (Table 2). Liposome forms spontaneously when lipids are dispersed in an aqueous medium. The thin-film hydration methods are simple and widely used in lab-scale and semiproduction scale operations. The liposome fabrication involves the three most important steps: vesicle formation, size reduction, and purification (Table 2).

\section{Liposome characterization}

Liposome behavior in both physical and biological systems is influenced by various parameters such as size, shape, lamellarity,

\begin{tabular}{|c|c|c|}
\hline Steps & Process & Types of Liposomes \\
\hline \multirow{7}{*}{$\begin{array}{l}\text { Vesicle } \\
\text { Formation }\end{array}$} & $\begin{array}{c}\text { Lipid hydration followed } \\
\text { by vortex or manual } \\
\text { stirring }\end{array}$ & $\begin{array}{c}\text { Multilamellar vesicles } \\
\text { (MLV) }\end{array}$ \\
\hline & $\begin{array}{l}\text { Reverse-phase } \\
\text { evaporation }\end{array}$ & $\begin{array}{l}\text { MLV, } \\
\text { Large unilamellar } \\
\text { vesicles (LUV) }\end{array}$ \\
\hline & Organic solvent injection & $\begin{array}{c}\text { MLV, LUV, Small } \\
\text { unilamellar vesicles } \\
\text { (SUV) }\end{array}$ \\
\hline & Freeze-thawing & MLV, LUV \\
\hline & $\mathrm{pH}$ gradient & LUV, SUV \\
\hline & Dehydration-rehydration & MLV \\
\hline & Detergent dialysis & MLV, LUV \\
\hline \multirow{4}{*}{$\begin{array}{l}\text { Vesicle } \\
\text { size-reduction }\end{array}$} & $\begin{array}{c}\text { Extrusion through } \\
\text { polycarbonate } \\
\text { membranes }\end{array}$ & LUV, SUV \\
\hline & $\begin{array}{c}\text { High-pressure } \\
\text { homogenization }\end{array}$ & LUV, SUV \\
\hline & Microfluidization & Mainly SUV \\
\hline & Sonication & Mainly SUV \\
\hline \multirow{4}{*}{ Purification } & Centrifugation & - \\
\hline & Dialysis & - \\
\hline & $\begin{array}{c}\text { Column chromatography } \\
\text { separation }\end{array}$ & - \\
\hline & Ultrafiltration & - \\
\hline
\end{tabular}

Table 2: Methods of liposome preparation [14-16].

entrapment volume, etc. Thus, liposome in-vivo behavior depends on these factors too (Table 3) [17].

\begin{tabular}{|c|c|}
\hline Characterization Parameters & Instrumentations \\
\hline \multicolumn{2}{|c|}{ Chemical Characterization } \\
\hline Drug Concentration by Assay & HPLC, UV \\
\hline $\begin{array}{l}\text { Phospholipid Concentration by } \\
\text { Barlett/Stewart assay }\end{array}$ & HPLC \\
\hline Cholesterol concentration & HPLC \\
\hline Lipid Peroxidation & $\begin{array}{c}\text { UV absorbance, TBA, iodometric, } \\
\text { GLC }\end{array}$ \\
\hline $\begin{array}{l}\text { Lipid hydrolysis, Cholesterol } \\
\text { auto-oxidation, ant- oxidant } \\
\text { degradation }\end{array}$ & HPLC, TLC, \\
\hline
\end{tabular}




\begin{tabular}{|c|c|}
\hline $\mathrm{pH}$ & pH meter \\
\hline Osmolarity & osmometer \\
\hline \multicolumn{2}{|l|}{ Physical Characterization } \\
\hline $\begin{array}{l}\text { Vesicle size and surface } \\
\text { morphology }\end{array}$ & $\begin{array}{l}\text { Transmission electron } \\
\text { microscopy (TEM), } \\
\text { Freeze-fracture electron } \\
\text { microscopy }\end{array}$ \\
\hline Size distribution & $\begin{array}{c}\text { Dynamic light scattering (DLS), } \\
\text { Zetasizer, TEM, Polymerase } \\
\text { chain reaction (PCR), } \\
\text { Gel permeation, Exclusion }\end{array}$ \\
\hline Surface charge & Free-flow electrophoresis \\
\hline $\begin{array}{l}\text { Electric surface potential and } \\
\mathrm{pH}\end{array}$ & $\begin{array}{c}\text { Zeta potential measurement, } \mathrm{pH} \\
\text { probes }\end{array}$ \\
\hline Lamellarity & SAXS, ${ }^{31} \mathrm{NMR}$, Freeze fracture EM \\
\hline Phase behavior & $\begin{array}{l}\text { Freeze fracture electronic } \\
\text { microscope, Differential } \\
\text { scanning calorimetry (DSC) }\end{array}$ \\
\hline$\%$ Entrapment Efficiency & $\begin{array}{l}\text { Minicolumn centrifugation, Gel } \\
\text { exclusion, Ion exchange, } \\
\text { Protamine aggregation, } \\
\text { Radiolabeling }\end{array}$ \\
\hline Drug release & Diffusion \\
\hline \multicolumn{2}{|l|}{ Biological Characterization } \\
\hline Sterility & Aerobic or anaerobic cultures \\
\hline Pyrogenicity & $\begin{array}{c}\text { Limulus amebocyte lysate (LAL) } \\
\text { test }\end{array}$ \\
\hline Animal toxicity & $\begin{array}{c}\text { Monitoring survival rates, } \\
\text { Histopathology }\end{array}$ \\
\hline
\end{tabular}

Table 3: Liposome Characterization [17].

\section{Conclusion}

Liposomal drug delivery is gaining popularity due to its contribution to the pharmaceutical drug delivery and cosmetics arena. In the pharmaceutical drug delivery sector, liposomes successfully footprinted in the commercial market in 1995 by introducing Doxil $^{\circledR}$. After that, the liposomal drug delivery system never looked back, and numerous researches are ongoing in both the academic and pharmaceutical industries. From this review, readers can get concise information on marketed liposomal drugs, preparation, and characterization.
Bibliography

1. Moinuddin S M., et al. "Facile formation of co-amorphous atenolol and hydrochlorothiazide mixtures via cryogenic-milling: enhanced physical stability, dissolution and pharmacokinetic profile". International Journal of Pharmaceutics 532.1 (2017): 393-400.

2. Moinuddin S M., et al. "Enhanced Physical Stability and Synchronized Release of Febuxostat and Indomethacin in Coamorphous Solids". AAPS PharmSciTech 21.2 (2020): 1-10.

3. Lombardino J G and Lowe J A. "The role of the medicinal chemist in drug discovery-then and now". Nature Reviews Drug Discovery 3.10 (2004): 853-862.

4. Kalepu S., et al. "Liposomal drug delivery system-a comprehensive review". International Journal of Drug Development and Research 5.4 (2013): 62-75.

5. Mishra H., et al. "A comprehensive review on Liposomes: a novel drug delivery system". Journal of Drug Delivery and Therapeutics 8.6 (2018): 400-404.

6. Bulbake U., et al. "Liposomal formulations in clinical use: an updated review". Pharmaceutics 9.2 (2017): 12.

7. Nambiar M., et al. "Post-COVID alliance-mucormycosis, a fatal sequel to the pandemic in India". Saudi Journal of Biological Sciences (2021).

8. DivakarPK.“FungalTaxa Responsible for Mucormycosis/“Black Fungus" among COVID-19 Patients in India". Journal of Fungi 7.8 (2021): 641.

9. Mucormycosis K K P. "A Black Fungus-Post COVID Complications". Journal of Regenerative Biology and Medicine 3.4 (2021): 1-8.

10. Rahman F I., et al. "Mucormycosis or black fungus infection is a new scare in South Asian countries during the COVID-19 pandemic: Associated risk factors and preventive measures". Journal of Medical Virology (2021).

11. Akbarzadeh A., et al. "Liposome: classification, preparation, and applications". Nanoscale Research Letters 8.1 (2013): 1-9.

12. Barba A A., et al. "Lipid delivery systems for nucleic-acidbased-drugs: From production to clinical applications". Pharmaceutics 11.8 (2019): 360.

13. Bangham A. "Lipid bilayers and biomembranes". Annual Review of Biochemistry 41.1 (1972): 753-776. 
14. New R and Liposomes, A. "Practical Approach". Rickwood D., Hames BM, ed. New York: IRL Press, Oxford University Press: 1990.

15. Vemuri S and Rhodes C. "Preparation and characterization of liposomes as therapeutic delivery systems: a review". Pharmaceutica Acta Helvetiae 70.2 (1995): 95-111.

16. de Araújo Lopes SC., et al. "Liposomes as carriers of anticancer drugs. Cancer treatment-conventional and innovative approaches" (2013).

17. Sharma Vijay K., et al. "Liposomes: present prospective and future challenges". International Journal of Current Pharmaceutical Review and Research 1.2 (2010): 6-16.

\section{Assets from publication with us}

- Prompt Acknowledgement after receiving the article

- Thorough Double blinded peer review

- Rapid Publication

- Issue of Publication Certificate

- High visibility of your Published work

Website: www.actascientific.com/

Submit Article: www.actascientific.com/submission.php

Email us: editor@actascientific.com

Contact us: +919182824667 